\title{
Bagaço de mandioca (Manihot esculenta, Crantz) na dieta de vacas leiteiras: consumo de nutrientes
}

\author{
[Cassava bagasse (Manihot esculenta, Crantz) in the diet of lactating cows: intake of nutrients] \\ L.P. Lima ${ }^{1}$, C.M. Veloso ${ }^{2}$, F.F. Silva ${ }^{2}$, P. Bonomo ${ }^{2}$, A.A. Pinheiro ${ }^{3,4}$, G.S. Dutra ${ }^{3,5}$, \\ I.G. Pereira Júnior ${ }^{3,5}$, J.M.C. Veloso ${ }^{3,5}$ \\ ${ }^{1}$ Zootecnia autônomo - Maiquinique, BA \\ ${ }^{2}$ Universidade Estadual do Sudoeste da Bahia - Itapetinga, BA \\ ${ }^{3}$ Aluno de graduação - UESB - Itapetinga, BA \\ ${ }^{4}$ Bolsista do CNPq \\ ${ }^{5}$ Bolsista da FAPESB
}

\begin{abstract}
RESUMO
Avaliou-se o efeito da inclusão de diferentes níveis $(0,5,10$ e 15\%) de bagaço de mandioca à dieta de 12 vacas mestiças leiteiras Holandês x Zebu (composição racial com variação de $1 / 4$ a 3/4 de sangue $\mathrm{H}$ x Z) com 478,5kg de peso corporal médio e com 100 a 150 dias de lactação, distribuídas em três Quadrados Latinos 4 x 4. Foi avaliado o consumo de matéria seca (MS), matéria orgânica (MO), proteína bruta (PB), extrato etéreo (EE), fibra em detergente neutro (FDN), fibra em detergente ácido (FDA), carboidratos totais $(\mathrm{CHT})$, carboidratos não-fibrosos $(\mathrm{CNF})$ e nutrientes digestíveis totais (NDT). Forneceu-se silagem de capim-elefante como fonte de volumoso. As relações volumoso:concentrado utilizadas foram de $65,19: 34,81 ; 61,59: 38,41 ; 59,08: 40,92$ e 54,76:45,24. Formularam-se as dietas isoprotéicas e isoenergéticas. Houve aumento linear do consumo de MS, MO, PB, CHT, CNF e NDT, efeito quadrático do consumo de EE e redução do consumo de FDA com o aumento do BM, enquanto o consumo de FDN não diferiu entre os tratamentos.O bagaço de mandioca pode ser utilizado até o nível de $15 \%$ de inclusão na dieta total de vacas mestiças leiteiras sem trazer transtornos fisiológicos ou nutricionais aos animais.

Palavras-chave: bovino de leite, bagaço de mandioca, consumo, resíduo, subproduto
\end{abstract}

\begin{abstract}
The effect of different inclusion levels $(0,5,10$, and 15\%) of cassava bagasse to the diet of 12 Holstein $x$ Zebu crossbred dairy cows (breed composition varying from 1/4 to $3 / 4 \mathrm{H} \times \mathrm{Z}$ blood), averaging $478.5 \mathrm{~kg}$ body weight and 100 to 150 days in milk was evaluated. Cows were distributed in three $4 \times 4$ latin squares. The intake of dry matter (DM), organic matter $(O M)$, crude protein $(C P)$, ether extract (EE), neutral detergent fiber (NDF), acid detergent fiber (ADF), total carbohydrates (TC), non fiber carbohydrates (NFC), and total digestible nutrients (TDN) were evaluated. Elephant grass silage was provided as roughage source. The roughage:concentrate ratios were 65.19:34.81; 61.59:38.41; 59.08:40.92; and 54.76:45.24. Isonitogen and isoenergetic diets were formulated. There was a linear increase in DM, OM, CP, TC, NFC, and TDN intakes, quadratic effect of EE intake, and a reduction of $A D F$ intake with the increase of the BM; while no diference among treatments was observed for NDF intake. The cassava bagasse can be used until 15\% inclusion level in the total diet of crossbred dairy cows without physiological or nutritional damage.
\end{abstract}

Keywords: cassava bagasse, dairy cattle, intake, residue, byproduct

Recebido em 3 de março de 2008

Aceito em 4 de julho de 2008

Endereço para correspondência (corresponding address)

Praça Aleixo Pereira Passos, 40 - 45770-000 - Maiquinique, BA

E-mail: leo.pl@hotmail.com 


\section{INTRODUÇÃO}

O consumo de nutrientes é um dos principais fatores associados ao desempenho animal, pois é determinante no atendimento das exigências de mantença e produção de ruminantes. Existem vários fatores relacionados ao consumo de alimento pelos bovinos, podendo este, ser limitado pelo alimento, pelo animal ou pelas condições de alimentação (Mertens, 1997). Nem sempre é possível atender às demandas de energia para animais de alta produção com IMS limitante, resultando em perda de peso e, conseqüentemente, redução na produção. A IMS depende de muitas variáveis, incluindo peso vivo, nível de produção de leite, estádio da lactação, condições ambientais, fatores psicogênicos e de manejo, histórico de alimentação, condição corporal e tipo e qualidade dos ingredientes da ração, particularmente forragens (Nutrient..., 1988).

Segundo o NRC, os valores mínimos de FDN na dieta de vacas são de 25 a $27 \%$, sendo que $75 \%$ desse total devem ser oriundos de forragens, para evitar problemas de redução nos teores de gordura do leite (Nutrient..., 1988).

O processamento da mandioca para produção de fécula gera vários subprodutos, como o bagaço que tem sido fornecido com resultados satisfatórios para bovinos por alguns criadores (Silveira, 1995). Esse subproduto possui, além de boa qualidade de fibra, um relativo teor de amido, mas os dados referentes à sua utilização por inclusão na dieta de vacas em lactação são limitados.
O objetivo deste trabalho foi avaliar os efeitos da inclusão de diferentes níveis de bagaço de mandioca à dieta de vacas mestiças leiteiras em lactação sobre o consumo dos nutrientes.

\section{MATERIAL E MÉTODOS}

Foram utilizadas 12 vacas mestiças Holandês x Zebu (composição racial variando de $1 / 4$ a $3 / 4$ de sangue $\mathrm{H} \times \mathrm{Z}$ ), apresentando em média $478,5 \mathrm{~kg}$ de peso corporal e com 100 a 150 dias de lactação ao início do período experimental, alojadas em baias individuais providas de cocho e bebedouro, nas dependências da Fazenda Água Azul no município de Macarani, BA.

As quatro dietas experimentais (Tab. 1) foram fornecidas aos animais respeitando-se $\mathrm{O}$ delineamento em triplo Quadrado Latino (QL) $4 \times 4$.

O volumoso utilizado foi silagem de capim-elefante (Pennisetum purpureum) obtido com plantas cortadas aos 70 dias e pré-secadas antes da ensilagem. O bagaço de mandioca, já seco, foi obtido em casas de farinha da região e sua inclusão à dieta foi feita pela sua adição aos concentrados nas devidas proporções. Os resultados das análises bromatológicas do volumoso, do bagaço de mandioca e dos concentrados estão contidos na Tab. 2. As dietas foram calculadas na tentativa de serem isoprotéicas e isoenergéticas e para conter nutrientes suficientes para mantença e produção de $15 \mathrm{~kg}$ de leite $/$ dia, de acordo com o NCR (Nutrient..., 2001).

Tabela 1. Dietas experimentais fornecidas a vacas leiteiras, na base da matéria seca

\begin{tabular}{lcccc}
\hline & \multicolumn{4}{c}{ Nível de inclusão de bagaço de mandioca (\%) } \\
\cline { 2 - 5 } V:C (MS) & 0 & 5 & 10 & 15 \\
MS(\%) & $65,19: 34,81$ & $61,59: 38,41$ & $59,08: 40,92$ & $54,76: 45,24$ \\
MO(\%) & 47,4 & 49,5 & 50,9 & 53,5 \\
PB(\%) & 90,8 & 91,1 & 91,4 & 91,5 \\
EE(\%) & 10,9 & 11,4 & 11,7 & 12,0 \\
FDN(\%) & 2,8 & 2,6 & 2,5 & 2,4 \\
FDA(\%) & 51,1 & 49,3 & 48,6 & 47,0 \\
CHOT(\%) & 21,5 & 20,7 & 19,7 & 19,5 \\
CNF(\%) & 77,1 & 77,2 & 77,2 & 77,1 \\
NDT(\%) & 26,1 & 27,9 & 28,6 & 30,0 \\
MM(\%) & 50,8 & 51,5 & 52,2 & 52,9 \\
\hline
\end{tabular}

V:C: relação volumoso:concentrado, MS: teores médios de matéria seca, MO: matéria orgânica, PB: proteína bruta, EE: extrato etéreo, FDN: fibra em detergente neutro, FDA: fibra em detergente ácido, CHOT: carboidratos totais, CNF: carboidratos não-fibrosos, NDT: nutrientes digestíveis totais, MM: matéria mineral. 
Tabela 2. Composição bromatológica da silagem de capim-elefante, do bagaço de mandioca e dos concentrados experimentais, na base da matéria seca

\begin{tabular}{lcccccc}
\hline \multirow{2}{*}{ Componente } & \multirow{2}{*}{$\begin{array}{c}\text { Silagem de } \\
\text { capim-elefante }\end{array}$} & \multirow{2}{*}{$\begin{array}{c}\text { Bagaço de } \\
\text { mandioca }\end{array}$} & \multicolumn{4}{c}{ \% de bagaço de mandioca na dieta } \\
\cline { 4 - 7 } MS & 25,67 & 87,5 & 88,17 & 87,83 & 87,62 & 87,16 \\
MO & 89,00 & 98,38 & 94,16 & 94,59 & 95,04 & 94,45 \\
PB & 4,66 & 1,95 & 22,50 & 22,10 & 21,80 & 20,90 \\
EE & 3,17 & 0,60 & 2,10 & 1,61 & 1,63 & 1,44 \\
MM & 11,00 & 1,62 & 5,84 & 5,41 & 4,96 & 5,55 \\
FDN & 72,26 & 12,02 & 11,39 & 12,54 & 14,49 & 16,49 \\
FDA & 28,76 & 6,73 & 7,84 & 7,47 & 6,67 & 8,34 \\
CNF & 8,75 & 85,06 & 58,2 & 58,3 & 57,1 & 55,6 \\
CHOT & 81,01 & 97,10 & 77,70 & 70,9 & 71,6 & 72,1 \\
NDT & 42,08 & 70,70 & 66,99 & 66,49 & 66,97 & 66,09 \\
\hline
\end{tabular}

MS: matéria seca, MO: matéria orgânica, PB: proteína bruta, EE: extrato etéreo, FDN: fibra em detergente neutro, FDA: fibra em detergente ácido, CHOT: carboidratos totais, CNF: carboidratos não fibrosos, NDT: nutrientes digestíveis totais, MM: matéria mineral.

$\mathrm{CNF}=100-(\mathrm{PB}+\mathrm{EE}+\mathrm{MM}+\mathrm{FDN}) ; \mathrm{CHOT}=\mathrm{FDN}+\mathrm{CNF}$.

NDT: de acordo com recomendação de Cappelle et al. (2001).

As vacas foram alimentadas duas vezes ao dia, às $6 \mathrm{~h}$ e $17 \mathrm{~h}$, com o alimento oferecido na forma de mistura completa, à vontade, de modo a permitir $5 \%$ de sobras. As quantidades oferecidas e de sobras foram registradas diariamente para estimativas do consumo.

O experimento foi constituído de quatro períodos experimentais, sendo o primeiro com duração de 16 dias: nove dias de adaptação e sete de coletas de dados, os três últimos períodos tiveram duração de 12 dias cada, sendo os primeiros sete dias considerados de adaptação, conforme recomendação de Oliveira (2000).

Os dados de consumo foram avaliados por análise de regressão por meio do SAEG (Sistema..., 2001), por intermédio de polinômios ortogonais e pela decomposição da soma do quadrado de tratamentos (níveis de inclusão de bagaço de mandioca) em efeitos linear, quadrático e cúbico, e os parâmetros testados pelo teste $\mathrm{F}$, com $1 \%$ de probabilidade.

\section{RESULTADOS E DISCUSSÃO}

Os dados de consumo de matéria seca (CMS), matéria orgânica $(\mathrm{CMO})$, proteína bruta $(\mathrm{CPB})$ e extrato etéreo (CEE), são apresentados na Tab. 3.

Os resultados da análise de regressão resultaram em equação linear crescente para CMS, CMO e CPB e equação quadrática para CEE, expressos em $\mathrm{kg} /$ dia, $\%$ PC e g/kg PM. O CMS com valores de 2,67 a
$3,35 \%$ do $\mathrm{PC}$, resultou em uma equação linear crescente, com a inclusão de bagaço de mandioca na dieta. $\mathrm{O}$ aumento da proporção de concentrado à medida que aumentou os níveis de bagaço de mandioca na dieta total foi responsável pelo aumento do CMS, CMO e CPB.

O aumento do CMS com o aumento da inclusão de bagaço de mandioca pode ter sido devido a maior ou mais rápida degradação ruminal do amido do bagaço de mandioca.

O consumo de MS foi influenciado pelo nível de bagaço de mandioca $(\mathrm{P}<0,01)$, diferente dos resultados encontrados por Silva et al. (2006) que ao utilizarem bagaço de mandioca, em diferentes níveis, na ensilagem de capim-elefante fornecida para novilhas leiteiras, observaram CMS de $5,5 \mathrm{~kg} / \mathrm{dia} ; 3,18 \% \mathrm{PC}$ e $115,42 \mathrm{~g} / \mathrm{kgPM}$, e que esse consumo não diferiu entre os tratamentos.

A média de CMS de 134,51 g/kgPM encontrada neste trabalho foi superior à média de $85,7 \mathrm{~g} / \mathrm{kgPM}$ observada por Ramos et al. (2000), que utilizaram bagaço de mandioca em substituição ao milho no concentrado para bovinos em crescimento, diferença essa que pode ser explicada pelo fato de esses autores terem utilizado uma categoria animal com exigências nutricionais diferentes. Esses autores observaram, também, que níveis acima de $48,74 \%$ de bagaço de mandioca no concentrado reduziram o CMS. 
Tabela 3. Médias e equações de regressão (ER) do consumo de matéria seca (MS), matéria orgânica $(\mathrm{MO})$, proteína bruta $(\mathrm{PB})$ e extrato etéreo (EE), em função do nível de bagaço de mandioca (BM) na dieta, e os respectivos coeficientes de determinação $\left(\mathrm{r}^{2}, \mathrm{R}^{2}\right)$ e de variação $(\mathrm{CV}, \%)$

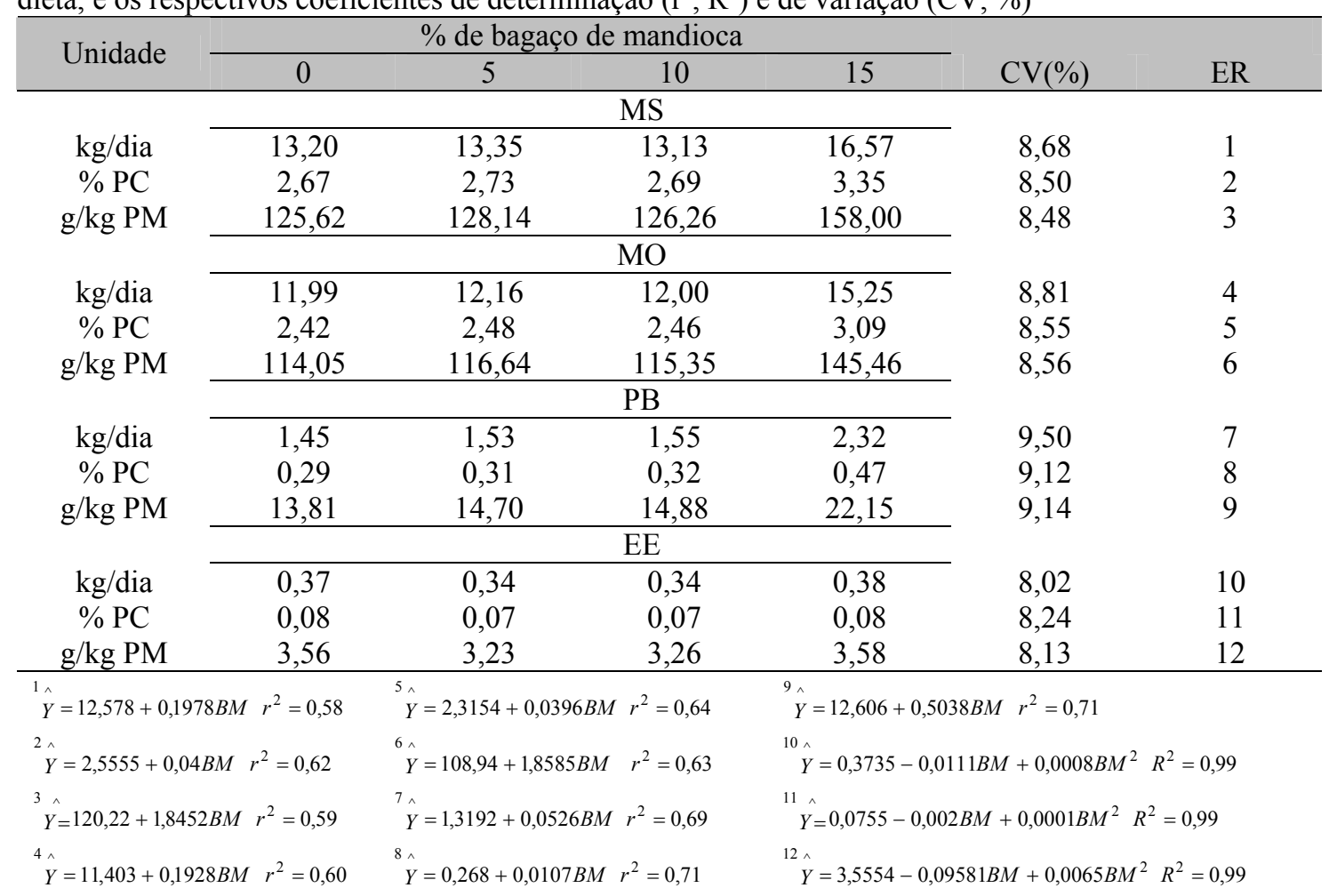

Diversos trabalhos citam reduções no CMS de rações com alguns resíduos da mandioca, atribuídas, em alguns casos, à pulverulência desses subprodutos. Neste estudo não foi possível observar se houve alguma interferência de algum aspecto físico do bagaço de mandioca ou de qualquer outro nutriente sobre o CMS.

O CMO, assim como o CMS, apresentou efeito linear positivo com a inclusão dos níveis de bagaço de mandioca. As médias variaram entre 11,99 e $15,25 \mathrm{~kg} / \mathrm{dia}, 2,42$ e $3,09 \%$ PC e 114,05 e $145,46 \mathrm{~g} / \mathrm{kgPM}$. Efeito semelhante foi observado por Figueira (2006) que, ao trabalhar com bovinos mestiços em confinamento, obteve médias que variaram entre 11,0 e $13,4 \mathrm{~kg} / \mathrm{dia} ; 2,9$ e $3,4 \% \mathrm{PC} ; 127,1$ e $150,4 \mathrm{~g} / \mathrm{kgPM}$.

$\mathrm{O}$ CPB, expresso em $\mathrm{kg} / \mathrm{dia}$, apresentou diferença significativa, resultando em efeito linear crescente em função do nível de inclusão de bagaço de mandioca $(\mathrm{P}<0,01)$. Com o aumento do nível de inclusão de bagaço de mandioca houve aumento no $\mathrm{CPB}$, devido ao maior consumo de MS.
A ingestão de PB também acompanhou o CMS, tanto em $\mathrm{kg} / \mathrm{dia}$ como em \%PC e g/kgPM. Devese atentar à possibilidade de a amônia, proveniente da uréia, ter sido prontamente utilizada pelos microrganismos ruminais na fermentação ruminal, possibilitando, assim, maior sincronismo entre a liberação de amônia e a fermentação dos carboidratos provenientes do bagaço de mandioca. Dessa forma, a digestão ocorreu mais rapidamente e permitiu maior CMS.

Médias inferiores de $\mathrm{CPB}$ em $\mathrm{g} / \mathrm{kgPM}$ foram verificadas por Ramos et al. (2000), que utilizaram bagaço de mandioca em substituição ao milho no concentrado para bovinos em crescimento. Essa diferença é devida, provavelmente, ao fato de terem trabalhado com uma categoria animal de menor exigência. A maior ingestão de proteína no nível de inclusão de $15 \%$ foi devida a maior concentração de PB na dieta total, como também a maior proporção de concentrado em relação ao volumoso. Podese, ainda, atribuir esse fato à seletividade 
daqueles animais, que tendem a consumir mais concentrado.

Para o CEE, foi detectada relação quadrática, com ponto mínimo de consumo em $7,3 \%$ de inclusão de bagaço de mandioca. $\mathrm{O}$ bagaço de mandioca apresenta menor teor de EE e sua inclusão reduz o EE na dieta total (Tab. 1). Figueira (2006) detectou efeito linear decrescente, exceto quando expresso em $\mathrm{kg} / \mathrm{dia}$. $\mathrm{O}$ autor atribuiu esse comportamento à diminuição dos teores de EE devido à incorporação de bagaço de mandioca na dieta total. O mesmo não aconteceu no presente estudo porque, mesmo elevando-se os níveis de bagaço de mandioca, os animais compensaram o deficit de EE consumindo maior quantidade de $\mathrm{e}$ concentrado, caracterizando, novamente, sua seletividade.

Os resultados obtidos para consumo de fibra em detergente neutro (CFDN), fibra em detergente ácido (CFDA), carboidratos totais (CCHOT), carboidratos não fibrosos (CCNF) e nutrientes digestíveis totais (CNDT) são apresentados na Tab. 4.

Tabela 4. Médias e equações de regressão (ER) do consumo de fibra em detergente neutro (FDN), fibra em detergente ácido (FDA), carboidratos totais (CHOT), carboidratos não fibrosos (CNF) e nutrientes digestíveis totais (NDT), em função do nível de bagaço de mandioca (BM) na dieta, e os respectivos coeficientes de determinação $\left(\mathrm{r}^{2}\right)$ e de variação $(\mathrm{CV}, \%)$

\begin{tabular}{|c|c|c|c|c|c|c|}
\hline \multirow{2}{*}{ Unidade } & \multicolumn{4}{|c|}{$\%$ de bagaço de mandioca } & \multirow[b]{2}{*}{$\mathrm{CV}(\%)$} & \multirow[b]{2}{*}{ ER } \\
\hline & 0 & 5 & 10 & 15 & & \\
\hline \multicolumn{7}{|c|}{ FDN } \\
\hline $\mathrm{kg} / \mathrm{dia}$ & 6,72 & 6,56 & 6,26 & 6,48 & 8,94 & 1 \\
\hline$\% \mathrm{PC}$ & 1,36 & 1,34 & 1,28 & 1,31 & 8,49 & 2 \\
\hline $\mathrm{g} / \mathrm{kgPM}$ & 63,93 & 62,92 & 60,15 & 61,79 & 8,54 & 3 \\
\hline & \multicolumn{6}{|c|}{ FDA } \\
\hline $\mathrm{kg} / \mathrm{dia}$ & 2,76 & 2,72 & 2,63 & 2,61 & 9,12 & 4 \\
\hline$\% \mathrm{PC}$ & 0,56 & 0,55 & 0,54 & 0,53 & 8,71 & 5 \\
\hline \multirow[t]{2}{*}{$\mathrm{g} / \mathrm{kgPM}$} & 26,32 & 26,06 & 25,32 & 24,91 & 8,75 & 6 \\
\hline & \multicolumn{6}{|c|}{ CHOT } \\
\hline $\mathrm{kg} / \mathrm{dia}$ & 10,14 & 10,27 & 10,11 & 12,56 & 8,76 & 7 \\
\hline$\% \mathrm{PC}$ & 2,05 & 2,10 & 2,07 & 2,54 & 8,51 & 8 \\
\hline \multirow[t]{2}{*}{$\mathrm{g} / \mathrm{kgPM}$} & 96,50 & 98,53 & 97,16 & 119,77 & 8,51 & 9 \\
\hline & \multicolumn{6}{|c|}{$\mathrm{CNF}$} \\
\hline $\mathrm{kg} / \mathrm{dia}$ & 3,47 & 3,76 & 3,79 & 5,92 & 9,80 & 10 \\
\hline$\% \mathrm{PC}$ & 0,72 & 0,75 & 0,78 & 1,21 & 11,95 & 11 \\
\hline \multirow[t]{2}{*}{$\mathrm{g} / \mathrm{kgPM}$} & 33,84 & 35,57 & 36,44 & 56,95 & 10,62 & 12 \\
\hline & \multicolumn{6}{|c|}{ NDT } \\
\hline $\mathrm{kg} / \mathrm{dia}$ & 6,74 & 6,91 & 6,90 & 9,28 & 8,78 & 13 \\
\hline$\% \mathrm{PC}$ & 1,36 & 1,41 & 1,41 & 1,88 & 8,64 & 14 \\
\hline $\mathrm{g} / \mathrm{kgPM}$ & 64,16 & 66,36 & 66,41 & 88,45 & 8,61 & 15 \\
\hline${ }^{1} \bar{Y}=6,51$ & \multicolumn{3}{|c|}{${ }^{6} \bar{Y}=25,65$} & \multicolumn{3}{|c|}{${ }^{11 \wedge}=1,2844+0,031 B M \quad r^{2}=0,68$} \\
\hline${ }^{2} \bar{Y}=1,32$ & \multicolumn{3}{|c|}{${ }^{7} \hat{Y}=9,7058+0,1418 B M \quad r^{2}=0,59$} & \multicolumn{3}{|c|}{${ }^{12} \hat{Y}=60,41+1,4581 B M \quad r^{2}=0,68$} \\
\hline${ }^{3}-\bar{Y}=62,20$ & \multicolumn{3}{|c|}{$\stackrel{8}{Y}=1,9707+0,0292 B M \quad r^{2}=0,63$} & \multicolumn{3}{|c|}{$267+0,1479 B M$} \\
\hline \multicolumn{2}{|l|}{$\stackrel{4}{Y}=2,7635$} & \multicolumn{2}{|c|}{${ }^{9} \hat{Y}=92,723+1,3688 B M \quad r^{2}=0,62$} & \multicolumn{3}{|c|}{ (a) } \\
\hline \multicolumn{2}{|l|}{$\stackrel{5}{Y}_{Y}=0,55$} & \multicolumn{2}{|c|}{${ }^{10} \hat{Y}=6,3173+0,152 B M \quad r^{2}=0,65$} & & $69+1,404 B$ & \\
\hline
\end{tabular}

O CFDN, expresso em $\mathrm{kg} / \mathrm{dia}$ \% $\% \mathrm{PC}$ e g/kg PM, não apresentou diferença significativa com o aumento do nível de bagaço de mandioca, devido à redução da FDN da dieta, pois, ao reduzir a proporção do volumoso que contém altos teores de fibra, elevou-se a quantidade de CNF da dieta.
O teor médio de FDN das dietas totais foi de $48,94 \%$, semelhante aos valores relatados por Silva et al. (2007) e bem acima dos valores (34\% e 38\%) sugeridos por Mertens (1997) para vacas com produção de 16 a $24 \mathrm{~kg}$ de leite corrigido para $4 \%$ de gordura. O teor médio de FDN da 
dieta está associado ao teor de FDN do volumoso (silagem de capim-elefante) de 72,26\%. Dessa forma, o CFDN em relação ao PC foi de 1,32\%, acima do valor de $1,2 \pm 0,1 \%$ PC sugerido por Mertens (1997), para se obter consumo ótimo de MS. No Brasil, vários autores encontraram consumos de FDN entre 1,3 e $1,6 \%$ do PC (Almeida, 1997; Campos, 1998; Moreira, 2000), o que pode ser uma adaptação dos animais às forrageiras tropicais.

Como a FDN geralmente fermenta e sua passagem pelo rúmen-retículo é mais lenta que os outros constituintes da dieta, podendo, assim, apresentar maior efeito de enchimento e ser considerada como limitadora da ingestão voluntária de MS. Neste trabalho, os teores de FDN da dieta podem ter influenciado o CMS das vacas em lactação.

O CFDA, expresso em kg/dia, apresentou comportamento linear decrescente com o aumento do nível de bagaço $(\mathrm{P}<0,01)$, devido à redução da FDA da dieta. Porém, quando expresso em \%PC e em $\mathrm{g} / \mathrm{kgPM}$ não foi detectada diferença no consumo, apenas decréscimo linear sem significado nutricional. Dias et al. (2008), trabalhando com novilhas leiteiras, também encontrou decréscimo linear no CFDA expresso em $\mathrm{kg} / \mathrm{dia}$ o qual, de forma semelhante ao presente estudo, variou a relação volumoso:concentrado, permitindo o ajuste energético da dieta.

O CCHOT apresentou efeito linear crescente $(\mathrm{P}<0,01)$ com a inclusão do bagaço de mandioca. Esse efeito pode ser explicado pelo aumento no CMS e da participação percentual do concentrado no nível mais alto de inclusão do bagaço.

O CCNF, conforme se elevou o nível de inclusão de bagaço de mandioca, apresentou aumento linear, pois o bagaço apresenta maior quantidade de CNF e, ao aumentar sua inclusão reduziu-se o teor de FDN. A diferença de CCNF entre as dietas pode ser atribuída à diferença no CMS.

Neste trabalho foram utilizadas dietas isoenergéticas, portanto, o CNDT aumentou com inclusão do bagaço de mandioca, pois o CNDT acompanhou diretamente o CMS. Figueira (2006), ao trabalhar com novilhos em crescimento, alimentados com bagaço de mandioca, também observou efeito linear crescente nas diferentes formas de expressão de CNDT.

\section{CONCLUSÕES}

Recomenda-se o uso de bagaço de mandioca na alimentação de vacas leiteiras mestiças até o nível de $15 \%$ de inclusão na dieta total. Mesmo havendo alterações no consumo de MS, MO, PB, CHT, CNF, NDT, EE e FDA, nenhum distúrbio nutricional ou fisiológico foi detectado.

\section{REFERÊNCIAS BIBLIOGRÁFICAS}

ALMEIDA, R.G. Saccharina em dietas para vacas lactantes. 1997. 52f. Dissertação (Mestrado) - Universidade Federal de Viçosa, Viçosa.

CAMPOS, J.M.S. Balanço dietético cátion-ânion na alimentação de vacas leiteiras, no período do pré-parto. 1998. 103f. Tese (Doutorado) Escola de Veterinária, Universidade Federal de Minas Gerais, Belo Horizonte.

CAPPELLE, E.R.; VALADARES FILHO, S.C.V.; SILVA, J.F.C. et al. Estimativas do valor energético a partir de características químicas e bromatológicas dos alimentos. R. Bras. Zootec, v.30, p.1837-1856, 2001.

DIAS, A.M.; SILVA, F.F.; VELOSO, C.M. et al. Bagaço de mandioca em dietas de novilhas leiteiras: consumo de nutrientes e desempenho produtivo. Arq. Bras. Med. Vet. Zootec., v.60, p.987-995, 2008.

FIGUEIRA, N.A. Bagaço de mandioca na alimentação de bovinos mestiços em confinamento. 2006. 66f. Dissertação (Mestrado) - Universidade Estadual do Sudoeste da Bahia, Vitória da Conquista.

MERTENS, D.R. Predicting in intake and digestibility using mathematical models of ruminal functions. J. Anim. Sci., v.64, p.15481558, 1997.

MOREIRA, A.L. Valor nutritivo de rações contendo silagem de milho e fenos de alfafa e de coast-cross como volumosos, para vacas lactantes e ovinos. 2000. 62f. Dissertação (Mestrado) - Universidade Federal de Viçosa, Viçosa. 
NUTRIENT requirements of dairy cattle. 6.ed. Washington: National Academic of Sciences, 1988. 157p.

NUTRIENT requirements of dairy cattle. 7.ed. National Academic Press, Washington: National Academic of Sciences, 2001. 408p

OLIVEIRA, A.S. Consumo, digestibilidade, produção e composição do leite, produção de proteína microbíana e estimativas das excreções de derivados de purinas e de uréia em vacas lactantes alimentadas com rações contendo diferentes teores de uréia. 2000. 98f. Dissertação (Mestrado) - Universidade Federal de Viçosa, Viçosa.

RAMOS, P.R.; PRATES, E.R.; FONTANELLI, R.S. et al. Uso de bagaço de mandioca em substituição ao milho no concentrado para bovinos em crescimento. Consumo de matéria seca, matéria orgânica e proteína bruta. R. Bras. Zootec, v.29, p.295-299, 2000.

SILVA, F.F.; AGUIAR, M.S.M.A.; VELOSO, C.M. et al. Desempenho de novilhas leiteiras alimentadas com silagem de capim-elefante com adição de diferentes níveis de bagaço de mandioca. Arq. Bras. Med. Vet. Zootec., v.58, p.205-211, 2006.

SILVA, F.F.; AGUIAR, M.S.M.A.; VELOSO, C.M. et al. Bagaço de mandioca na ensilagem do capim-elefante: qualidade das silagens e digestibilidade dos nutrientes. Arq. Bras. Med. Vet. Zootec., v.59, p.719-729, 2007.

SILVEIRA, J.A. Tem resíduos virando carne. Granja, v.51, p.41-42, 2005.

SISTEMA de análises estatísticas - SAEG. Viçosa: UFV, 2001. 301p. 